\title{
DETERMINAÇÃO DE Cd E Pb: AVALIAÇÃo DE SEDIMENTOS DO RIO JUNDIAÍ - SP E RIBEIRÃo PIRAÍ - SP E LODO PROVENIENTE DE UMA ESTAÇÃO DE TRATAMENTO DE ESGOTOS
}

\author{
Silvéria Neves de Paula e Souza, Pedro Sérgio Fadini e Edenir Rodrigues Pereira-Filho* \\ Departamento de Química, Universidade Federal de São Carlos, Rodovia Washington Luiz, km 235, 13565-905, São Carlos - SP, \\ Brasil
}

Recebido em 11/12/08; aceito em 13/5/09; publicado na web em 6/11/09

\begin{abstract}
Cd AND Pb DETERMINATION: EVALUATION OF SEDIMENTS FROM JUNDIAÍ AND PIRAI STREAMS AND SLUDGE FROM A WASTEWATER TREATMENT PLANT. Jundiaí river is the main stream in the Jundiaí Hydrographic Basin and its water is not considered safe for public supply. The water problem is getting worse with the development of the region. The Pirai stream, have been one of the last potable water resource. With the purpose of investigating the sediment quality, due to its influence on the water quality, bioavailable and total $\mathrm{Cd}$ and $\mathrm{Pb}$ were determined using TS-FF-AAS. Total $\mathrm{Cd}$ and $\mathrm{Pb}$ up to 2.47 and $24.7 \mu \mathrm{g} \mathrm{g}{ }^{-1}$ were measured, respectively. The wastewater sludge showed concentrations of 4.01 and $171 \mu \mathrm{g} \mathrm{g}^{-1}$, for the same metals.
\end{abstract}

Keywords: extraction; atomic absorption spectrometry; sediments.

\section{INTRODUÇÃO}

No século XXI a escassez de água para consumo populacional é um problema presente em diversas regiões do planeta. ${ }^{1}$ No estado de São Paulo (Brasil), por exemplo, com uma população por volta de 40 milhões de habitantes, já é uma realidade a procura por fontes alternativas de água para suprir cidades, além do estabelecimento de estratégias de reuso.

Um exemplo de região com baixa disponibilidade hídrica é a Bacia Hidrográfica do Rio Jundiaí que possui uma área de $1.200 \mathrm{~km}^{2}$ e uma população de cerca da 1 milhão de habitantes, distribuída em seis cidades cortadas pelo referido rio. Um importante manancial desta bacia hidrográfica é o Ribeirão Piraí situado na fronteira entre as cidades de Indaiatuba e Salto, ambas localizadas a $100 \mathrm{~km}$ da cidade de São Paulo, com 180 mil e 100 mil habitantes, respectivamente. Por enquanto, a qualidade da água deste rio foi atestada como apropriada para abastecimento, após tratamento, de acordo com a resolução CONAMA no 357. Um cenário da qualidade de um corpo hídrico pode ser inferido pela avaliação de seus sedimentos, pois estes possuem capacidade para transportar e acumular elementos tóxicos como $\mathrm{Cd}$ e $\mathrm{Pb}$, entre outros. ${ }^{2} \mathrm{~A}$ avaliação de elementos químicos tóxicos em diferentes compartimentos e matrizes ambientais permite avaliações de risco com respeito à ecotoxicologia e à saúde humana. ${ }^{3}$

A expansão da atividade industrial, a modificação dos padrões de consumo da população e as consequentes demandas por energia e recursos minerais, em associação com políticas ambientais inadequadas, incipientes ou ausentes, têm colaborado para o aumento da concentração de espécies químicas deletérias nos diversos compartimentos ambientais, seja pela introdução de moléculas sintéticas ou mobilizações a partir de reservatórios naturais. Um exemplo clássico é o lançamento de carbono originalmente fóssil, na atmosfera, sob a forma de $\mathrm{CO}_{2}$. Neste contexto, a população de centros urbanos tem convivido com corpos aquáticos contaminados e com a movimentação geoquímica e espacial destes contaminantes provenientes tanto de fontes difusas como localizadas, ${ }^{4}$ bem como produzido vários tipos de resíduos, dentre eles o lodo oriundo de estações de tratamento de esgotos. ${ }^{5}$

Dentre os elementos tóxicos mais comumente encontrados no ambiente, destacam-se $\mathrm{Cd}$ e $\mathrm{Pb}$. Estes metais podem ser incorporados ao organismo humano pela ingestão de alimentos e/ou água, a inalação

*e-mail: erpf@ufscar.br de ar contaminado e exposição a um específico local de trabalho. ${ }^{6} \mathrm{~A}$ contaminação por estes metais é um problema público em diversos países, uma vez que eles podem provocar distúrbios relacionados à saúde, incluindo danos aos rins, fígado, sistema vascular, aumento da pressão arterial e desordens metabólicas. ${ }^{7}$ A concentração destes metais em diferentes matrizes ambientais é ditada pelo grau de contaminação natural ou antrópica do ambiente estudado, sendo que é necessária a busca e aplicação de técnicas analíticas sensíveis para a determinação de teores de metais, com valores, algumas vezes, da ordem de sub $\mu \mathrm{g} \mathrm{kg}^{-1}$.

Gáspár e Berndt ${ }^{8}$ apresentaram, em 2000, uma técnica analítica denominada espectrometria de absorção atômica com tubo na chama e aerossol térmico (TS-FF-AAS, Thermospray Flame Furnace Atomic Absorption Spectrometry) e algumas aplicações e suas características principais são listadas na Tabela 1 , onde pode ser verificada a versatilidade da TS-FF-AAS, com abrangência de aplicações desde amostras biológicas a alimentos. A TS-FF-AAS emprega um tubo metálico como atomizador localizado sobre uma chama de ar/acetileno e as amostras são introduzidas com o auxílio de um capilar cerâmico (tipicamente com 0,5 $\mathrm{mm}$ d.i.). Utilizando esta proposta, é alcançado um longo tempo de residência dos átomos na chama possibilitando a total introdução da amostra. Como resultado, os limites de detecção (LD) podem alcançar, em alguns casos, a mesma magnitude observada para a espectrometria de absorção atômica com forno de grafite (GFAAS, Graphite Furnace AAS).

Embora com recursos hídricos estratégicos e limitantes para o desenvolvimento regional, a Bacia Hidrográfica do Rio Jundiaí tem ainda poucos resultados analíticos divulgados, fazendo com que este trabalho contribua para gerar subsídios para a avaliação do impacto ambiental ao qual tem sido submetida, como também demonstrar mais uma vez a potencialidade do emprego da TS-FF-AAS, como uma técnica analítica rápida, viável e precisa para a determinação de metais em matrizes ambientais.

\section{PARTE EXPERIMENTAL}

\section{Amostragem e pré-tratamento das amostras}

As amostras de sedimentos foram coletadas em agosto e setembro de 2006 em diversos pontos ao longo da Bacia do Rio Jundiaí, a jusante da cidade de Itupeva, com auxílio de canos de 
Tabela 1. Trabalhos empregando TS-FF-AAS e suas principais características

\begin{tabular}{|c|c|c|}
\hline Amostra(s) & LD (Analito) & Observações \\
\hline Alimentícias ${ }^{9}$ & $\mathrm{Cd}, \mathrm{Cu}, \mathrm{Mn}, \mathrm{Pb}, \mathrm{Zn}$ & Estudo de tubos atomizadores de Ni e Ti em TS-FF-AAS. \\
\hline Aquosas $^{8}$ & $0,19(\mathrm{Cd}), 1,3(\mathrm{Cu}), 5,2(\mathrm{Tl}), 13(\mathrm{~Pb})$ e $21 \mu \mathrm{g} \mathrm{L}^{-1}(\mathrm{Hg})$ & Primeiro trabalho sobre a TS-FF-AAS. \\
\hline Aquosas $^{10}$ & $69 \mu \mathrm{g} \mathrm{L}^{-1}(\mathrm{~Pb})$ & Transporte da amostra com o uso de um gás. \\
\hline Aquosas $^{11}$ & $0,2(\mathrm{Zn})$ e $310 \mu \mathrm{g} \mathrm{L}^{-1}(\mathrm{Se})$ & Aumento no poder de detecção para 17 elementos. \\
\hline Aquosas $^{12}$ & $1,7 \mathrm{mg} \mathrm{L}^{-1}(\mathrm{Sn})$ & Determinação de $\mathrm{Sn}$ como alternativa à chama $\mathrm{C}_{2} \mathrm{H}_{2} / \mathrm{N}_{2} \mathrm{O}$. \\
\hline Aquosas $^{13}$ & $\mathrm{Cd}$ & Utilização de imagens do aerosol térmico. \\
\hline Aquosas $^{14}$ & $7,0 \mu \mathrm{g} \mathrm{L}^{-1}(\mathrm{Co})$ & Utilização de derivatização para a determinação de Co. \\
\hline Bebidas $^{15}$ & 2,2 (Cu), $18(\mathrm{Mn}), 1,6(\mathrm{~Pb}), 0,9 \mu \mathrm{g} \mathrm{L} \mathrm{L}^{-1}(\mathrm{Zn})$ & Introdução direta das amostras nos atomizadores. \\
\hline Biológicas ${ }^{16}$ & $2,1 \mu \mathrm{g} \mathrm{L} \mathrm{L}^{-1}(\mathrm{Co})$ & Utilização de extração por ponto nuvem. \\
\hline Biológicas $^{17}$ & $8,7 \mu \mathrm{g} \mathrm{L} \mathrm{L}^{-1}(\mathrm{Se})$ & O LD foi 95 vezes menor quando comparado com FAAS. \\
\hline $\mathrm{CRM}^{\prime} \mathrm{s}^{18}$ & $0,007(\mathrm{Cd}), 0,17 \mu \mathrm{g} \mathrm{L}^{-1}(\mathrm{~Pb})$ & Separação e pré-concentração em coluna $\mathrm{C} 18$. \\
\hline $\mathrm{CRM}^{\prime} \mathrm{s}^{19}$ & $0,03 \mu \mathrm{g} \mathrm{L}^{-1}(\mathrm{Cd})$ & Pré-concentração utilizando bagaço de uva. \\
\hline $\mathrm{CRM}^{\prime} \mathrm{s}^{20}$ & $0,2 \mu g \mathrm{~L}^{-1}(\mathrm{Ag})$ & $\begin{array}{l}\text { Utilização de dois procedimentos de extração por ponto } \\
\text { nuvem. }\end{array}$ \\
\hline Alimentícias ${ }^{21}$ & $0,5(\mathrm{Cd}), 4,3(\mathrm{Cu})$ e $3,5 \mu \mathrm{g} \mathrm{L}-1(\mathrm{~Pb})$ & Utilização de amostras na forma de uma suspensão. \\
\hline Alimentícias ${ }^{22}$ & $0,12 \mu \mathrm{g} \mathrm{L}^{-1}(\mathrm{Cd})$ & Pré-concentração em linha. \\
\hline Alimentícias $^{23}$ & $0,1(\mathrm{Cd})$ e $2,4 \mu \mathrm{g} \mathrm{L} \mathrm{L}^{-1}(\mathrm{~Pb})$ & Utilização de CRM para testes de exatidão. \\
\hline Alimentícias ${ }^{24}$ & $0,005(\mathrm{Cd})$ e $0,1 \mu \mathrm{g} \mathrm{L}^{-1}(\mathrm{~Pb})$ & Utilização de CRM para testes de exatidão. \\
\hline Alimentícias $^{25}$ & $2,2(\mathrm{Cu})$ e $0,91 \mu \mathrm{g} \mathrm{L} \mathrm{L}^{-1}(\mathrm{Zn})$ & Testes de adição e recuperação. \\
\hline Medicinais $^{26}$ & $0,9 \mu \mathrm{g} \mathrm{L} \mathrm{L}^{-1}(\mathrm{Cd})$ & Utilização de mini-frascos para o preparo das amostras. \\
\hline Ambientais $^{27}$ & $0,2(\mathrm{Cd})$ e $8 \mu \mathrm{g} \mathrm{L}^{-1}(\mathrm{~Pb})$ & As amostras foram introduzidas na forma de suspensão. \\
\hline Vegetais ${ }^{28}$ & $8 \mathrm{ng} \mathrm{L}^{-1}(\mathrm{Cd})$ & Extração em fase sólida. \\
\hline Aquosas $^{29}$ & $0,22 \mu \mathrm{g} \mathrm{dm}^{-3}(\mathrm{Cu})$ & $\begin{array}{l}\text { Utilização de planejamento fatorial na otimização do procedi- } \\
\text { mento de pré-concentração. }\end{array}$ \\
\hline Águas e cigarro ${ }^{30}$ & $11,4 \mathrm{ng} \mathrm{L}^{-1}(\mathrm{Cd})$ & A exatidão foi avaliada com testes de adição e recuperação. \\
\hline Ambientais $^{31}$ & $0,32(\mathrm{Cd}), 26(\mathrm{~Pb})$ e $0,21 \mu \mathrm{g} \mathrm{L}^{-1}(\mathrm{Zn})$ & $\begin{array}{l}\text { A exatidão foi avaliada com amostras certificadas de referên- } \\
\text { cia. }\end{array}$ \\
\hline Água e urina ${ }^{32}$ & $0,04 \mu \mathrm{g} \mathrm{L}^{-1}(\mathrm{Cd})$ & Utilização de extração por ponto nuvem. \\
\hline Bebidas $^{33}$ & $12,9(\mathrm{Cu}), 1,8(\mathrm{Cd}), 5,3 \mu \mathrm{g} \mathrm{L} \mathrm{L}^{-1}(\mathrm{~Pb})$ & Utilização do método de adição de padrões. \\
\hline
\end{tabular}

PVC, com diâmetro de 2 polegadas, que se mostraram eficientes, tanto para a retirada de perfis, quanto de amostras superficiais. A Figura 1 mostra algumas características importantes da região estudada e os pontos de coleta (ver quadrados). Nove amostras (de 1 a 9) foram coletadas no Rio Jundiaí e 5 (amostras 10 a 14) no Ribeirão Piraí, enquadrado como classe 2 (ver linha pontilhada na Figura 1) de acordo com a Resolução CONAMA no $357 .{ }^{34}$ Isso significa que suas águas podem, dentre outros fins, também servir para o abastecimento da população. O Rio Jundiái é um rio bastante impactado e enquadrado como classe 3 (ver linha sólida na Figura 1) na cidade de Jundiaí. Suas águas, de acordo com a Resolução CONAMA no 357 , podem ser utilizadas para o abastecimento para consumo humano, após tratamento convencional ou avançado, e outro fins menos nobres. Nas proximidades da cidade de Itupeva, este rio passa a ser enquadrado como classe 4 (ver linha tracejada na Figura 1), com água apropriada apenas para navegação e harmonia paisagística. Além das amostras de sedimentos, foi estudada uma amostra de lodo de esgoto. Esta amostra (amostra 15) foi coletada na Estação de Tratamento de Esgotos da Companhia Saneamento de Jundiaí, denominada de ETE-CSJ, após o mesmo ter sido gerado em um sistema de lagoas aeradas, dragado a partir de uma lagoa de

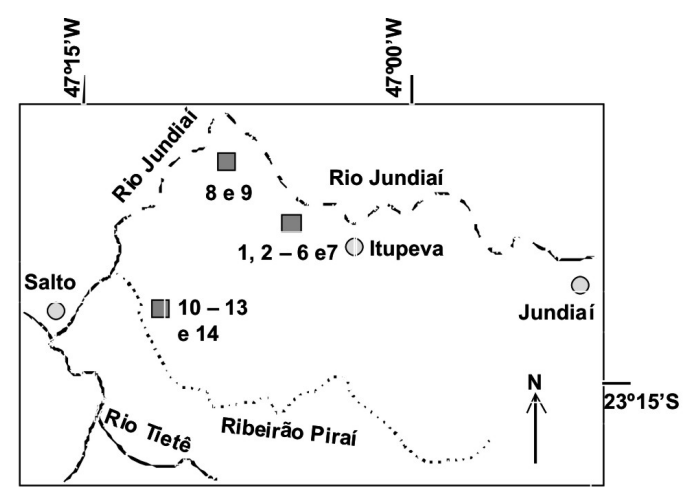

Figura 1. Descrição da região estudada: pontos de coleta e características dos rios. Os círculos representam as cidades (Jundiaí, Itupeva e Salto) ao longo do Rio Jundiaí e Ribeirão Piraí, já os quadrados representam os pontos de coleta. As linhas sólidas, tracejada e pontilhada representam os rios Classe 3,4 e 2 , respectivamente

sedimentação e submetido a tratamento físico-químico com polímeros, deságue em centrífuga e secagem em pátio. Esta estação recebe 
esgoto industrial e doméstico de Jundiaí, com vazão próxima a 1 $\mathrm{m}^{3} \mathrm{~s}^{-1}$ com DQO (Demanda química de oxigênio) e DBO (Demanda bioquímica de oxigênio) de 1.500 e $800 \mathrm{mg} \mathrm{O}_{2} \mathrm{~L}^{-1}$, respectivamente, e opera com $97 \%$ de eficiência média avaliada em termos de remoção da DBO. ${ }^{35} \mathrm{~A}$ motivação para avaliação desta matriz é que este tipo de lodo é normalmente candidato à destinação agrícola.

As amostras foram secas em estufa $\left(65^{\circ} \mathrm{C}\right)$ até massa constante. Após este procedimento foram trituradas com uso de almofariz e pistilo até atingir tamanho de partícula da ordem de $212 \mu \mathrm{m}$.

O método proposto foi validado com os seguintes materiais certificados: lodo de esgoto (CRM'S 2782 e 146R) e sedimento do rio Buffalo (CRM 8704). Para as amostras certificadas e não certificadas, o número de réplicas autênticas foi igual a 3 .

\section{Instrumentação}

Para as determinações de $\mathrm{Cd}$ e $\mathrm{Pb}$ nas amostras de sedimentos foi utilizado um espectrômetro de absorção atômica com chama Varian SpetrAA-640, no qual se adaptaram os componentes da TS-FF-AAS. Este equipamento possui uma lâmpada de deutério para correção da radiação de fundo. Nas determinações foi empregada uma chama de ar/acetileno. Outros parâmetros operacionais, tais como, taxas de fluxo dos gases e corrente da lâmpada foram aqueles recomendados pelo fabricante do equipamento. Todos os componentes do sistema TS são mostrados na Figura 2. Neste sistema é empregado um tubo de $\mathrm{Ni}$ (99\% de pureza) ajustado sobre o queimador da chama de ar/ acetileno, com o auxílio de um suporte de aço. As dimensões do tubo foram iguais a $100 \mathrm{~mm}$ de comprimento com 10 e $12 \mathrm{~mm}$ de diâmetros interno e externo, respectivamente. Uma particularidade do tubo é a presença de 6 orifícios circulares na parte inferior $(2 \mathrm{~mm}$ de diâmetro), para permitir a entrada parcial da chama de ar/acetileno e outro orifício circular na parte frontal (2,5 mm de diâmetro) para conectar o capilar cerâmico (Friatec, Alemanha) com $0,5 \mathrm{~mm}$ de diâmetro interno, 2,0 mm de diâmetro externo, comprimento de $10 \mathrm{~cm}$ e composto por $\mathrm{Al}_{2} \mathrm{O}_{3}(99 \%)$. Para a formação do aerossol térmico é necessário introduzir o capilar cerâmico cerca de $2 \mathrm{~mm}$ no interior do tubo de $\mathrm{Ni}$. As soluções foram coletadas com o auxílio de um injetor comutador confeccionado em acrílico e acoplado a uma bomba peristáltica (Ismatec, modelo MCP, Suíça).

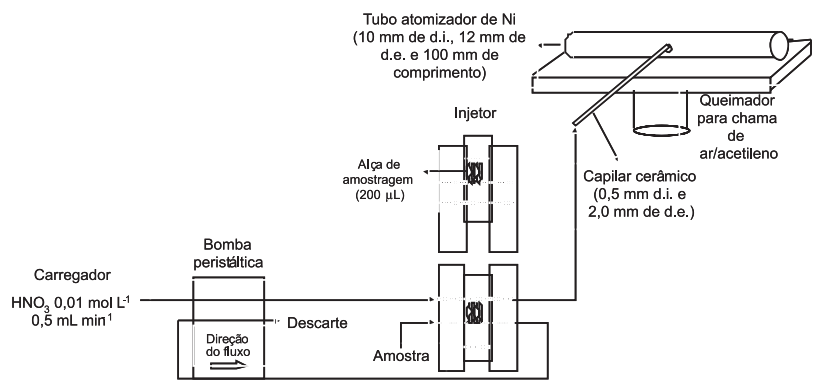

Figura 2. Descrição do sistema TS-FF-AAS composto por bomba peristáltica, injetor comutador confeccionado em acrílico, capilar cerâmico com 0,5 mm de d.i. e 2,0 mm de d.e. e tubo de $\mathrm{Ni}(99 \%)$ atomizador localizado sobre o queimador de uma chama de ar/acetileno

O volume de amostra, branco e soluções padrão foi de $200 \mu \mathrm{L}$ e introduzido na linha do carregador (solução de $\mathrm{HNO}_{3} 0,01 \mathrm{~mol}$ $\mathrm{L}^{-1}$ ou solução de EDTA $0,5 \%$ (m/v)/Triton X-100 0,1\% (v/v)) com uma vazão de $0,5 \mathrm{~mL} \mathrm{~min}^{-1}$ (Figura 2). Os sinais transientes foram registrados durante $100 \mathrm{~s}$ e, posteriormente, organizados em uma planilha de dados. Devido às altas concentrações de $\mathrm{Cd}$ e $\mathrm{Pb}$ avaliadas na amostra de lodo de esgoto, esta foi analisada utilizando FAAS.

\section{Materiais e reagentes}

As vidrarias e materiais utilizados para o preparo das amostras, soluções padrões e soluções carregadoras foram previamente deixados em solução de $\mathrm{HCl} \mathrm{10 \%} \mathrm{v/v} \mathrm{durante} 12 \mathrm{~h}$, posteriormente foram lavados com água destilada/deionizada. Todos os reagentes (Merck, Darmstadt, Alemanha) utilizados foram de grau analítico. As soluções padrões foram preparadas a partir de diluições sucessivas da solução estoque $1000 \mathrm{mg} \mathrm{L}^{-1}$. Para as determinações utilizando a TS-FF-AAS, os valores de concentração da curva analítica de $\mathrm{Cd}$ e $\mathrm{Pb}$ variaram entre 2 a 100 e entre 100 a $500 \mu \mathrm{g} \mathrm{L}^{-1}$, respectivamente. No caso da FAAS, as curvas de calibração utilizadas variaram de 0,1 a 3,0 mg $\mathrm{L}^{-1}$ para $\mathrm{Cd}$ e de 0,5 a $6,0 \mathrm{mg} \mathrm{L}^{-1}$ para $\mathrm{Pb}$. Em ambos os casos, as soluções foram preparadas com $\mathrm{HNO}_{3} 0,01 \mathrm{~mol} \mathrm{~L}{ }^{-1}$.

\section{Preparo das amostras e determinação das concentrações biodisponíveis e totais de $\mathrm{Cd}$ e $\mathbf{P b}$}

Para a extração das concentrações biodisponíveis de $\mathrm{Cd}$ e $\mathrm{Pb}$ foi utilizado um procedimento desenvolvido por Mozeto et al.,${ }^{36}$ no qual é recomendado que sejam adicionados $25 \mathrm{~mL}$ de uma solução de $\mathrm{HCl}$ $0,1 \mathrm{~mol} \mathrm{~L}^{-1}$ a 1,000 $\mathrm{g}$ de amostra e depois os frascos foram colocados em um agitador (Thermoline Maxi-Mix III, modelo 65800, EUA) por $2 \mathrm{~h}$. A suspensão obtida foi filtrada e somente o sobrenadante foi analisado.

As concentrações totais de $\mathrm{Cd}$ e $\mathrm{Pb}$ foram determinadas após o seguinte procedimento de preparação: $0,100 \mathrm{~g}$ das amostras foram pesadas em frascos de Teflon, foram adicionados $2,0 \mathrm{~mL}$ de uma mistura contendo $\mathrm{HNO}_{3}-\mathrm{HCl}(1: 3 \mathrm{v} / \mathrm{v})$ e $1,0 \mathrm{ml} \mathrm{de} \mathrm{H}_{2} \mathrm{O}_{2}$. As amostras foram pré-digeridas por $12 \mathrm{~h}$, depois os frascos de Teflon foram fechados e colocados em um forno de micro-ondas (Milestone, ETHOS 1, Itália). O programa de aquecimento foi o seguinte: estágio $1-400$ W, 3 min; estágio $2-850 \mathrm{~W}, 6$ min; estágio $3-1000 \mathrm{~W}, 10 \mathrm{~min}$ e, estágio $4-0 \mathrm{~W}, 15 \mathrm{~min}$.

\section{RESULTADOS E DISCUSSÃO}

\section{Concentrações biodisponíveis e totais de $\mathrm{Cd}$ e $\mathbf{P b}$}

A Figura 3 mostra alguns sinais analíticos para $\mathrm{Cd}$ obtidos por TS-FF-AAS. Com a ajuda da Figura 3 é possível verificar uma ampla faixa analítica com sinais variando de 2 a $100 \mu \mathrm{g} \mathrm{L}^{-1} \mathrm{e}$ a repetibilidade dos sinais foi cerca de $3 \%$.

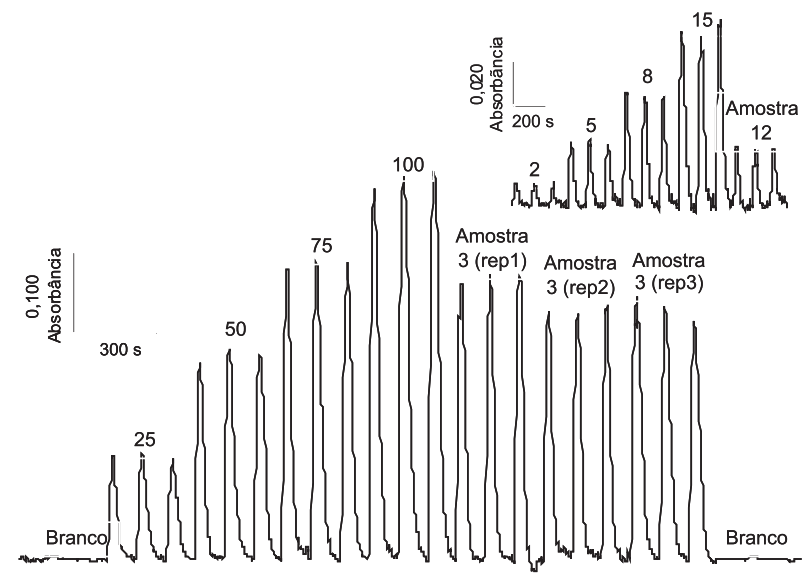

Figura 3. Sinais analíticos de Cd para soluções padrões de 2 a $100 \mu \mathrm{g} \mathrm{L}^{-1}$ e amostras 3 e 12 (ver detalhes na Tabela 2). Os números sobre os sinais representam as concentrações de Cd e a identificação das amostras 
Os LD's para $\mathrm{Cd}$ e $\mathrm{Pb}$ foram 1,1 e $17 \mu \mathrm{g} \mathrm{L}^{-1}$, respectivamente, e os limites de quantificação (LQ) foram 3,8 e $55 \mu \mathrm{g} \mathrm{L}^{-1}$ para $\mathrm{Cd}$ e $\mathrm{Pb}$, respectivamente. Estas figuras de mérito foram calculadas multiplicando-se o desvio padrão do branco da curva por 3 (no caso do LD) e por 10 (no caso do LQ) e dividindo-se o valor obtido pela inclinação da reta da curva analítica. ${ }^{37}$

A Tabela 2 mostra as concentrações biodisponíveis e totais de $\mathrm{Cd}$ e $\mathrm{Pb}$ para as 14 amostras de sedimento, para a amostra de lodo (15) e para as amostras certificadas de lodo de esgoto e sedimento de rio. As amostras de 1 a 9 são relacionadas ao Rio Jundiaí (Figura 1). A primeira amostra (1) foi coletada em um lago formado por uma extração irregular de areia. Esta extração foi embargada por uma decisão judicial e para recuperar o local foram ali depositados resíduos de construção civil e, inadequadamente, também resíduos industriais. Este histórico explica as altas concentrações biodisponíveis encontradas neste local: $1,70 \mu \mathrm{g} \mathrm{g}^{-1}$ para $\mathrm{Cd}$ e 13,6 $\mu \mathrm{g} \mathrm{g} \mathrm{g}^{-1}$ para $\mathrm{Pb}$. As amostras de 2 a 6 são relacionadas a um testemunho de sedimento (de 0 a 38 $\mathrm{cm})$ do rio Jundiaí. Em média, a concentração biodisponível de $\mathrm{Cd}$ nestas amostras foi 17 vezes maior do que aquelas encontradas no Ribeirão Piraí (testemunho de sedimento de 0 a $90 \mathrm{~cm}$ - amostras de 10 a 14). No caso do $\mathrm{Pb}$, as determinações de concentrações biodisponíveis nestes perfis apresentaram praticamente os mesmos valores, em toda a extensão do testemunho.

A amostra 7 foi coletada na correnteza entre o rio Jundiaí e a lagoa descrita anteriormente, e as concentrações biodisponíveis foram 0,84 (Cd) e 9,28 $\mu \mathrm{g} \mathrm{g}^{-1}(\mathrm{~Pb})$.

As demais amostras do Rio Jundiaí (8 e 9) apresentaram concentrações biodisponíveis de 0,84 e 0,62 (Cd) e 6,93 e 5,99 $\mu \mathrm{g} \mathrm{g}^{-1}(\mathrm{~Pb})$.
A amostra 14 apresentou características bastante arenosas e está relacionada ao fundo do Ribeirão Piraí apresentando uma concentração de $\mathrm{Pb}$ de $2,91 \mu \mathrm{g} \mathrm{g}^{-1}$. As concentrações de $\mathrm{Cd}$ nas amostras após o preparo para as determinações biodisponíveis e total ficaram abaixo do LD.

Durante a determinação das concentrações totais dos metais por TS-FF-AAS foi observado um constante entupimento do capilar cerâmico (ver detalhes na Figura 2). Este entupimento foi devido à deposição de sais ou óxidos na extremidade do capilar. Neste caso, as determinações de $\mathrm{Cd}$ e $\mathrm{Pb}$ nas amostras digeridas só foram possíveis após a substituição do carregador de $\mathrm{HNO}_{3} 0,01 \mathrm{~mol} \mathrm{~L}^{-1}$ por uma mistura contendo $0,5 \%(\mathrm{~m} / \mathrm{v})$ de EDTA e $0,1 \%(\mathrm{v} / \mathrm{v})$ de Triton X-100. Uma possível explicação para o bom desempenho desta mistura é que o EDTA forma complexos com os concomitantes cationicos presentes nas amostras, enquanto que o Triton X-100 efetua uma limpeza das paredes internas do capilar reduzindo a deposição de sais e óxidos em sua extremidade. O desempenho desta mistura de EDTA e Triton X-100 foi bastante satisfatório e não foram testadas concentrações altas de $\mathrm{HNO}_{3}$ como carregador. Altas concentrações de $\mathrm{HNO}_{3}$ podem acarretar em dois problemas: rápida deterioração do tubo de $\mathrm{Ni}$ e, problemas com alta intensidade da radiação de fundo, devido à formação de $\mathrm{NO}_{\mathrm{x}}$ no interior do tubo. ${ }^{38}$

As maiores concentrações totais foram observadas na amostra 1:2,47 $\mu \mathrm{g} \mathrm{g}^{-1}$ de $\mathrm{Cd}$ e 24,7 $\mu \mathrm{g} \mathrm{g}^{-1}$ de $\mathrm{Pb}$. As concentrações totais de $\mathrm{Cd}$ foram 3 vezes maiores no testemunho de sedimento do Rio Jundiaí (amostras de 2 a 6) quando comparado com o testemunho de sedimento do Ribeirão Piraí (amostras de 10 a 14). A concentração de $\mathrm{Pb}$ foi similar para ambos os perfis amostrados.

Tabela 2. Concentrações $\left(\mu \mathrm{g} \mathrm{g}^{-1}\right)$ biodisponíveis e totais de $\mathrm{Cd}$ e $\mathrm{Pb}(\mathrm{n}=3)$

\begin{tabular}{|c|c|c|c|c|c|}
\hline \multirow{2}{*}{ Amostra } & \multicolumn{2}{|c|}{$\mathrm{Cd}\left(\mu \mathrm{g} \mathrm{g}^{-1}\right)$} & \multicolumn{2}{|c|}{$\mathrm{Pb}\left(\mu \mathrm{g} \mathrm{g}^{-1}\right)$} & \multirow{2}{*}{ Observação } \\
\hline & Biodisponível & Total & Biodisponível & Total & \\
\hline 1 & $1,70 \pm 0,11$ & $2,47 \pm 0,44$ & $13,6 \pm 0,9$ & $24,7 \pm 3,3$ & \multirow{7}{*}{$\begin{array}{c}S=23^{\circ} 08,169^{\prime} \\
W=47^{\circ} 06,493\end{array}$} \\
\hline $2^{\mathrm{a}}$ & $1,36 \pm 0,19$ & $1,64 \pm 0,30$ & $8,67 \pm 0,94$ & $15,0 \pm 1,9$ & \\
\hline $3^{\mathrm{a}}$ & $1,62 \pm 0,15$ & $1,55 \pm 0,07$ & $9,06 \pm 0,71$ & $12,7 \pm 3,7$ & \\
\hline $4^{\mathrm{a}}$ & $1,53 \pm 0,07$ & $1,70 \pm 0,14$ & $9,04 \pm 1,22$ & $19,1 \pm 1,8$ & \\
\hline $5^{\mathrm{a}}$ & $1,49 \pm 0,26$ & $1,41 \pm 0,15$ & $10,1 \pm 0,5$ & $9,3 \pm 2,2$ & \\
\hline $6^{\mathrm{a}}$ & $1,40 \pm 0,22$ & $1,42 \pm 0,20$ & $9,06 \pm 0,66$ & $19,7 \pm 6,6$ & \\
\hline 7 & $0,84 \pm 0,11$ & $1,22 \pm 0,11$ & $9,28 \pm 1,22$ & $16,8 \pm 1,9$ & \\
\hline 8 & $0,84 \pm 0,05$ & $1,87 \pm 0,24$ & $6,93 \pm 0,44$ & $19,0 \pm 4,0$ & \multirow{3}{*}{$\begin{array}{r}\mathrm{S}=23^{\circ} 07,140 \\
\mathrm{~W}=47^{\circ} 11,965\end{array}$} \\
\hline 9 & $0,62 \pm 0,04$ & $0,86 \pm 0,18$ & $5,99 \pm 0,71$ & $31,0 \pm 2,1$ & \\
\hline $10^{\mathrm{b}}$ & $0,08 \pm 0,01$ & $0,58 \pm 0,30$ & $6,54 \pm 0,48$ & $13,2 \pm 4,4$ & \\
\hline $11^{\mathrm{b}}$ & $0,08 \pm 0,01$ & $0,55 \pm 0,10$ & $7,17 \pm 0,58$ & $23,9 \pm 2,5$ & \multirow{3}{*}{$\begin{array}{l}\mathrm{S}=23^{\circ} 11,017^{\prime} \\
\mathrm{W}=47^{\circ} 14,786\end{array}$} \\
\hline $12^{\mathrm{b}}$ & $0,09 \pm 0,01$ & $0,44 \pm 0,13$ & $8,68 \pm 0,31$ & $9,8 \pm 0,4$ & \\
\hline $13^{\mathrm{b}}$ & $0,09 \pm 0,01$ & $0,48 \pm 0,12$ & $7,68 \pm 0,43$ & $8,9 \pm 2,6$ & \\
\hline $14^{\mathrm{c}}$ & $\mathrm{ND}^{\mathrm{h}}$ & $\mathrm{ND}^{\mathrm{h}}$ & $2,91 \pm 0,44$ & $11,1 \pm 2,2$ & \\
\hline $15^{\mathrm{d}}$ & $5,41 \pm 0,04$ & $4,01 \pm 0,71$ & $71,0 \pm 2,0$ & $171 \pm 5$ & - \\
\hline CRM 2782 & - & $2,80 \pm 0,36(67)^{\mathrm{i}}$ & - & $\begin{array}{l}538 \pm 12 \\
\quad(94)^{\mathrm{i}}\end{array}$ & - \\
\hline CRM 146R & - & $17,1 \pm 0,9(91)^{\mathrm{i}}$ & - & $602 \pm 16(99)^{\mathrm{i}}$ & - \\
\hline CRM 8704" & - & $2,98 \pm 0,41(101)^{\mathrm{i}}$ & - & $121 \pm 2(81)^{\mathrm{i}}$ & - \\
\hline
\end{tabular}

${ }^{a}$ Testemunho de sedimento de 0 a $38 \mathrm{~cm}$. ${ }^{\text {b}}$ Testemunho de sedimento de 0 a $90 \mathrm{~cm}$. ${ }^{\mathrm{c}} \mathrm{Fundo}$ do rio. ${ }^{\mathrm{d}}$ Amostra de lodo de esgoto. ${ }^{\mathrm{e}}$ Industrial Sludge (CRM 2782 ): valores certificados iguais a $4,17 \pm 0,09 \mu \mathrm{g} \mathrm{g}^{-1}(\mathrm{Cd})$ e $574 \pm 11 \mu \mathrm{g} \mathrm{g}^{-1}(\mathrm{~Pb})$. ${ }^{\mathrm{f}}$ Industrial Sludge (CRM 146R): valores certificados iguais a $18,8 \pm 0,5 \mu \mathrm{g} \mathrm{g} \mathrm{g}^{-1}(\mathrm{Cd})$ e $609 \pm 15 \mu \mathrm{g} \mathrm{g}^{-1}(\mathrm{~Pb}) .{ }^{\mathrm{g}}$ Buffalo river sediment (CRM 8704): valores certificados iguais a 2,94 $\pm 0,29 \mu \mathrm{g} \mathrm{g}^{-1}(\mathrm{Cd})$ e $150 \pm 17 \mu \mathrm{g} \mathrm{g}{ }^{-1}(\mathrm{~Pb}) .{ }^{\mathrm{h}} \mathrm{Não}$ detectado. ${ }^{i} \mathrm{Re}-$ cuperações observadas $(\%)$. 
As concentrações totais de $\mathrm{Cd}$ e $\mathrm{Pb}$ na amostra 7 foram 1,22 e $16,8 \mu \mathrm{g} \mathrm{g}^{-1}$, respectivamente. Para as amostras 8 e 9 estas concentrações foram 1,87 e $0,86 \mu \mathrm{g} \mathrm{g}^{-1}$ para Cd e 19,0 e 31,0 $\mu \mathrm{g} \mathrm{g}^{-1}$ para $\mathrm{Pb}$, respectivamente. $\mathrm{Na}$ amostra 14 não foi detectado $\mathrm{Cd}$. Para $\mathrm{Pb}$, a concentração total foi $11,1 \mu \mathrm{g} \mathrm{g}^{-1}$. O Cd foi o melhor indicador de poluição na área estudada, devido ao fato de que a sua concentração nas amostras do Rio Jundiaí (área mais poluída) foi mais alta quando comparada com os valores observados no Ribeirão Piraí (Tabela 2). Este comportamento pode ser verificado na Figura 4, onde é mostrada uma boa correlação entre a concentração total e a biodisponível para o Cd. As amostras dentro do círculo pontilhado (Figura 4) foram coletadas no Ribeirão Piraí (amostras de 10 a 14), e apresentaram as menores concentrações de $\mathrm{Cd}$. No caso do $\mathrm{Pb}$ não foi possível estabelecer uma relação entre as concentrações totais e biodisponíveis (Figura 5), muito provavelmente em função dos teores basais de $\mathrm{Pb}$ serem significativamente maiores do que os de $\mathrm{Cd}$, o que faz com que pequenos aportes de $\mathrm{Pb}$ não sejam facilmente detectáveis. Acrescentam-se ainda os fatos de que o $\mathrm{Pb}$ foi largamente disperso na atmosfera durante o período em que foi utilizado com antidetonante na gasolina, na forma de chumbo tetraetila, ${ }^{39} \mathrm{e}$ incorporado em defensivos agrícolas na forma de arseniato de chumbo. ${ }^{40}$

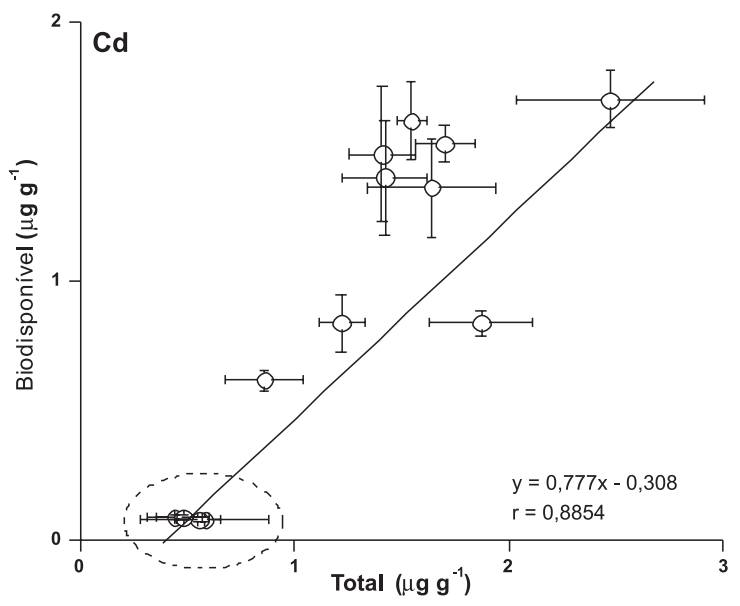

Figura 4. Concentração total versus concentração biodisponível $\left(\mu g g^{-1}\right)$ de Cd nas amostras estudadas. Os círculos dentro da elipse pontilhada representam as amostras provenientes do Ribeirão Piraí (amostras de 10 a 14). A equação do lado direito mostra a equação da reta relacionando as concentrações totais e biodisponíveis

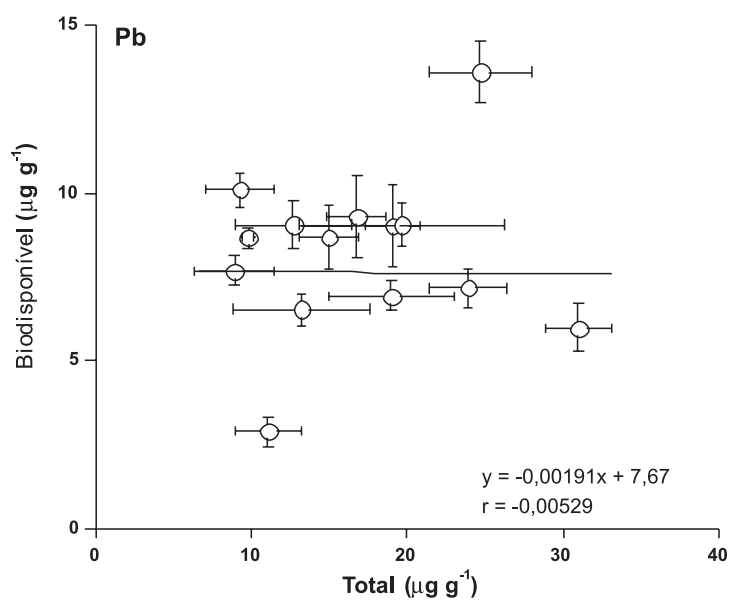

Figura 5. Concentração total versus concentração biodisponível $\left(\mu g g^{-1}\right)$ de Pb nas amostras estudadas. A equação do lado direito mostra a equação da reta relacionando as concentrações totais e biodisponiveis
Para o lodo de esgoto, as concentrações biodisponíveis foram 5,41 e 71,0 $\mathrm{mg} \mathrm{g}^{-1}$ para $\mathrm{Cd}$ e $\mathrm{Pb}$, respectivamente. As concentrações totais para estes metais foram 4,01 $\mathrm{mg} \mathrm{kg}^{-1}$ para Cd e $171 \mathrm{mg} \mathrm{kg}^{-1}$ para $\mathrm{Pb}$. Os limites de detecção para Cd $\left(63 \mu \mathrm{g} \mathrm{L}^{-1}\right)$ e $\mathrm{Pb}\left(119 \mu \mathrm{g} \mathrm{L}^{-1}\right)$ utilizando FAAS foram cerca de 60 e 30 vezes maiores, respectivamente, do que aqueles observados com TS-FF-AAS. Já os limites de quantificação utilizando FAAS foram 210 e $395 \mu \mathrm{g} \mathrm{L}^{-1}$ para Cd e $\mathrm{Pb}$, respectivamente.

De acordo com a resolução CONAMA n ${ }^{\circ} 375$, que estabelece os níveis máximos de metais tóxicos (39 e $300 \mu \mathrm{g} \mathrm{g}^{-1}$ para $\mathrm{Cd}$ e $\mathrm{Pb}$, respectivamente), a amostra 15 poderia ser utilizada na agricultura para fins de fertilização do solo. Entretanto, outros testes biológicos e físico-químicos necessitam ser efetuados para a utilização segura e controlada da aplicação do lodo de esgoto.

A exatidão do método proposto foi avaliada com a análise das amostras certificadas de lodo de esgoto (CRM 2782), lodo industrial (CRM 146R) e sedimento do Rio Búfalo (CRM 8704). As recuperações obtidas situaram-se entre 67 e $101 \%$ para o Cd e entre 81 e $99 \%$ para o $\mathrm{Pb}$. Mesmo com recuperações de até $67 \%$ a preocupação ambiental com o Cd foi caracterizada. Esta baixa recuperação é devido ao preparo de uma amostra relativamente complexa. As concentrações de $\mathrm{Cd}$ e $\mathrm{Pb}$ obtidas para as amostras certificadas também são apresentadas na Tabela 2 .

\section{CONCLUSÃO}

O Ribeirão Piraí é considerado um corpo aquático passível de ter suas águas captadas, para fins de abastecimento, uma vez que é enquadrado como Classe 2, de acordo com a Resolução CONAMA no 357 . O cenário ambiental atual da região denota preocupação em função de que no Ribeirão Piraí, a montante dos pontos amostrados, já na cidade de Cabreúva, tem sido grande a ocupação dos solos por residências e também o seu uso para atividades agrícolas e de criação de animais ao longo de toda a sua extensão. Neste contexto, a avaliação da qualidade dos sedimentos constitui-se num parâmetro indicador da qualidade das águas. Com os resultados obtidos é possível inferir, de modo comparativo, que o Ribeirão Piraí, por apresentar concentrações de Cd biodisponível significativamente menores do que o observado para o Rio Jundiaí, confirma a sua potencialidade para abastecimento, o que só pode ser atestado, após o tratamento da água e verificação de adequação à Portaria no 518 do Ministério da Saúde, que estabelece os padrões de potabilidade para águas de abastecimento no Brasil. A importância da avaliação de espécies químicas no sedimento reside no fato de que a concentração, por exemplo, de metais neste compartimento pode ser até algumas ordens de magnitude maior do que na coluna d'água, permitindo a detecção de problemas emergentes, antes da ocorrência de um impacto mensurável no recurso hídrico. Neste contexto, a TS-FF-AAS mostrou uma grande potencialidade em trabalhos de avaliação e/ou monitoramento ambiental, por conciliar velocidade analítica, baixo custo e sensibilidade quando comparada à atomização em forno de grafite, que apresenta limites de detecção similares.

\section{AGRADECIMENTOS}

À Coordenação de Aperfeiçoamento de Pessoal de Nível Superior (CAPES) pela bolsa de pesquisa concedida a S. N. P. Souza, ao Conselho Nacional de Desenvolvimento Científico e Tecnológico (CNPq - Processos números 484396/2006-4 e 308834/2006-2) e à Fundação de Amparo à Pesquisa do Estado de São Paulo (FAPESP) pelo suporte financeiro (processos número 04/01970-4 e 07/04515-4). 


\section{REFERÊNCIAS}

1. Stikker, A.; Futures 1998, 30, 43.

2. Mozeto, A. A.; Umbuzeiro, G.A.; Jardim, W.F.; Métodos de coleta, análises físico-químicas e ensaios biológios e ecotoxicológicos de sedimentos de água doce, 1ª. ed., Editora Cubo: São Carlos, 1996.

3. Davydova, S.; Microchem. J. 2005, 79, 133.

4. Filgueiras, A. V.; Lavilla, I.; Bendicho, C.; Sci. Total Environ. 2004, 330, 115.

5. Yaman, M.; Bakirdere, S.; Microchim. Acta 2003, 141, 47.

6. Robson, M.; Ecotox. Envirol. Safe 2003, 56, 104.

7. Vahter, M.; Akesson, A.; Lidén, C.; Cecatelli, S.; Berglund, M.; Environ. Res. 2007, 104, 85 .

8. Gáspár, A.; Berndt, H.; Spectrochim. Acta B 2000, 55, 587.

9. Petrucelli, G. A.; Stocco, P. K.; Bueno, M. I. M. S.; Pereira-Filho, E. R.; J. Anal. Atom. Spectrom. 2006, 21, 1298.

10. Gáspár, A.; Széles, E.; Berndt, H.; Anal. Bional. Chem. 2002, 372, 136.

11. Davies, J.; Berndt, H.; Anal. Chim. Acta 2003, 479, 215.

12. Lobo, F. A.; Villafranca, A. C.; Oliveira, A. P.; Moraes, M.; Ecl. Quim. 2005, 30, 61 .

13. Brancalion, M. L.; Sabadini, E.; Arruda, M. A. Z.; Anal. Chem. 2007, 79, 6527.

14. Matos, G. D.; Arruda, M. A. Z.; Talanta 2008, 76, 475.

15. Nascentes, C. C.; Kamogawa, M. Y.; Fernandes, K. G.; Arruda, M. A. Z.; Nogueira, A. R.; Nóbrega, J. A.; Spectrochim. Acta B 2005, 60, 749.

16. Donati, G. L.; Nascentes, C. C.; Nogueira, A. R. A.; Arruda, M. A. Z.; Nóbrega, J. A.; Microchem. J. 2006, 82, 189.

17. Rosini, F.; Nascentes, C. C.; Neira, J. Y.; Nóbrega, J. A.; Talanta 2007, 73,845 .

18. Ivanova, E.; Berndt, H.; Pulvermacher, E.; J. Anal. Atom. Spectrom. 2004, 19, 1507.

19. Matos, G. D.; Arruda, M. A. Z.; Spectrosc. Lett. 2006, 39, 755.

20. Wu, P.; Gao, Y.; Cheng, G.; Yang, W.; Lv, Y.; Hou, X.; J. Anal. Atom. Spectrom. 2008, 23, 752 .
21. Pereira-Filho, E. R.; Berndt, H.; Arruda, M. A. Z.; J. Anal. Atom. Spectrom. 2002, 17, 1308.

22. Tarley, C. R. T.; Arruda, M. A. Z.; Anal. Sci. 2004, 20, 961.

23. Pereira, M. G.; Pereira-Filho, E. R.; Berndt, H.; Arruda, M. A. Z.; Spectrochim. Acta B 2004, 59, 515.

24. Berndt, H.; Pulvermacher, E.; Anal. Bional. Chem. 2005, 382, 1826.

25. Nascentes, C. C.; Arruda, M. A. Z.; Nogueira, A. R.; Nóbrega, J. A.; Talanta 2004, 64, 912

26. Brancalion, M. L.; Arruda, M. A. Z.; Microchim. Acta 2005, 150, 283.

27. Petrucelli G. A.; Poppi, R. J.; Mincato, R. L.; Pereira-Filho, E. R.; Talanta 2007, 71, 620 .

28. Amorim, F. A.; Bezerra, M. A.; Microchim. Acta 2007, 159, 183.

29. Tarley, C. R. T.; Figueiredo, E. C.; Matos, G. D.; Anal. Sci. 2005, 21, 1337.

30. Tarley, C. R. T.; Barbosa, A. F.; Segatelli, M. G.; Figueiredo, E. C.; Luccas, P. O.; J. Anal. Atom. Spectrom. 2006, 21, 1305.

31. González, E.; Ahumada, R.; Medina, V.; Quim. Nova 2004, 27, 873.

32. Wu, P.; Zhang, Y.; Lv, Y.; Hou, X.; Spectrochim. Acta Part B 2006, 61, 1310 .

33. Schiavo, D.; Neira, J. Y.; Nóbrega, J. A.; Talanta 2008, 76, 1113.

34. Resolução CONAMA no 357; Ministério do Meio Ambiente, http:// www.mma.gov.br/port/conama/legiabre.cfm?codlegi=459, acessada em Abril 2008

35. Fadini, P. S.; Alciati, J. C.; Barros, J. C. C.; Guimarães, J. R.; RMZ Materials and Geoenvironment 2004, 51, 83.

36. Mozeto, A. A.; Silvério, P. F.; Soares, A.; Sci. Total Environ. 2001, 266, 135.

37. Analytical Methods Committee; Analyst 1987, 112, 199.

38. Todolí, J-L.; Mermet, J-M.; Spectrochim. Acta Part B 1999, 54, 895.

39. Bacon, J. R.; Jones, K. C.; McGrath, S. P.; Johnston, A. E.; Environ. Sci. Technol. 1996, 30, 2511.

40. Davenport, J. R.; Peryea, F. J.; Water, Air, Soil Pollut. 1991, 57-58, 101. 